\title{
DYNAMICS OF ANTIBODY LEVELS: ASYMPTOTIC PROPERTIES
}

\author{
KATARZYNA PICHÓR AND RYSZARD RUDNICKI
}

\begin{abstract}
We study properties of a piecewise deterministic Markov process modeling the changes in concentration of specific antibodies. The evolution of densities of the process is described by a stochastic semigroup. The long-time behaviour of this semigroup is studied. In particular we prove theorems on its asymptotic stability.
\end{abstract}

\section{INTRODUCTION}

In [4] the authors introduced a mathematical model of the immune system. The immune status is the concentration of specific antibodies, which appear after infection with a pathogen and remain in serum, providing protection against future attacks of that same pathogen. Over time the number of antibodies decreases until the next infection. During fighting the invader the immunity is boosted and then the immunity is gradually waning, etc. Thus the concentration of antibodies is described by a stochastic process whose trajectories are decreasing functions $x(t)$ between subsequent infections. These functions satisfy the differential equation

$$
x^{\prime}(t)=g(x(t)) .
$$

It is assumed that the time it takes the immune system to clear infection is negligible and that if $x$ is the concentration of antibodies at the moment of infection, then $Q(x)>x$ is the concentration of antibodies just after clearance of infection. An explicit expression for $Q$ was derived in [5, 16]. It is also assumed that the moments of infections are independent of the state of the immune system and they are distributed according to a Poisson process $\left(N_{t}\right)_{t \geq 0}$ with rate $\Lambda>0$.

This research was partially supported by the National Science Centre (Poland) Grant No. 2017/27/B/ST1/00100.

2010 Mathematics Subject Classification. Primary: 47D06; Secondary: 35Q92; 60J75; $92 \mathrm{D} 30$.

Key words and phrases. Immune status, physiologically structured population, stochastic semigroup, asymptotic stability, flow with jumps. 


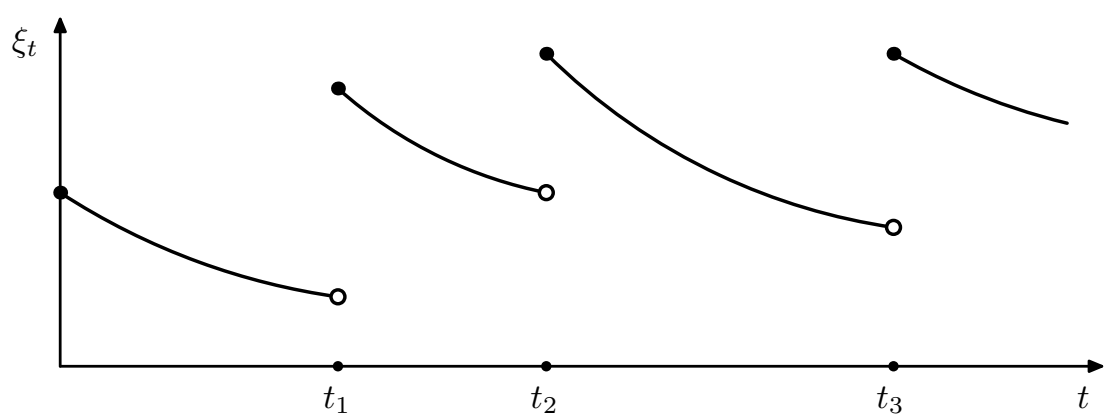

Figure 1. An example of a trajectory of the process $\xi_{t}$.

The immune status is a flow on the interval $[0, \infty)$ with jumps at random moments $t_{0}<t_{1}<t_{2}<\ldots$ (see Fig. 1). Such a flow belongs to the family of piecewise deterministic Markov processes [3, 15]. We denote this process by $\left(\xi_{t}\right)_{t \geq 0}$ and it is defined by the following equations

$$
\xi_{t_{n}}=Q\left(\xi_{t_{n-1}^{-}}\right), \quad \xi_{t}^{\prime}=g\left(\xi_{t}\right) \text { for } t \in\left[t_{n-1}, t_{n}\right), \quad N_{t_{n}}=N_{t_{n}^{-}}+1=n .
$$

It means that the process $\left(\xi_{t}\right)_{t \geq 0}$ satisfies the following stochastic differential equation

$$
d \xi_{t}=g\left(\xi_{t}\right) d t+\left(Q\left(\xi_{t}\right)-\xi_{t}\right) d N_{t}
$$

One of the main interesting problems is the evolution of the distribution of this process, in particular the existence of a unique stationary density $f^{*}$ and its asymptotic stability. It is worth to mention that if the process $\left(\xi_{t}\right)_{t \geq 0}$ has a unique stationary density $f^{*}$ then, according to the ergodic theorem, $f^{*}$ is the density of distribution of the immune status in the population. In [4] the asymptotic stability of a stationary density $f^{*}$ was proved for a function $Q$ which is unimodal and has properties: $\lim _{x \rightarrow 0} Q(x)=\infty$ and $\lim _{x \rightarrow \infty}(Q(x)-x)=$ const.

The aim of this note is to show that asymptotic stability holds for a large class of $C^{1}$-functions $Q$. In particular we extend the result from the paper [4] to the significant case when the increase of the concentration of antibodies after the infection is bounded. Moreover, we present another technique to prove this result, which seems to be easier in applications because it does not require to prove directly the existence of an invariant density. The main idea of the paper is to formulate the problem in the terms of stochastic semigroups and then apply some results concerning the Foguel alternative [11, 12], which 
gives conditions when a stochastic semigroup is asymptotically stable or sweeping.

The organization of the paper is as follows. In section 2 we present the assumptions concerning our model and formulate the main problem in the terms of stochastic semigroups. Section 3 contains the definitions and results concerning asymptotic properties of stochastic semigroups and the proof of the main result of the paper. In the last section we discuss the case when concentration of antibodies is bounded and we give some examples.

\section{A SEMigroup FORMUlation of the PROBLEM}

Concerning $g$ and $Q$ we assume the following

(A1) $g:[0, \infty) \rightarrow \mathbb{R}$ is a $C^{1}$-function such that $g(x)<0$ for $x>0$ and $g(0)=0$

(A2) $Q:[0, \infty) \rightarrow(0, \infty)$ is a $C^{1}$-function such that $Q(x)>x$ for $x \geq 0$,

(A3) $|A|=0 \Longrightarrow\left|Q^{-1}(A)\right|=0$, where $A$ is a Borel subset of $[0, \infty)$ and $|\cdot|$ denotes the Lebesgue measure.

We denote by $\pi_{t} x_{0}$ the solution $x(t)$ of Eq. (1) with the initial condition $x(0)=x_{0} \geq 0$.

Assumption (A3) allows us to introduce [7, 14] a linear operator $P_{Q}$ on the space $L^{1}=L^{1}[0, \infty)$ given by the formula

$$
\int_{A} P_{Q} f(x) d x=\int_{Q^{-1}(A)} f(x) d x
$$

for each $f \in L^{1}$ and all Borel subsets $A$ of $[0, \infty)$. The operator $P_{Q}$ is called the Frobenius-Perron operator for the transformation $Q$. The adjoint of the Frobenius-Perron operator $P^{*}: L^{\infty}[0, \infty) \rightarrow L^{\infty}[0, \infty)$ is given by $P^{*} g(x)=g(Q(x))$ and it is called the Koopman operator or the composition operator.

Denote by $D$ the subset of the space $L^{1}$ which contains all densities

$$
D=\left\{f \in L^{1}: f \geq 0,\|f\|=1\right\} .
$$

The Frobenius-Perron operator describes the evolution of densities under the action of the transformation $Q$ and it is an example of a stochastic or Markov operator, which is defined as a linear operator $P: L^{1} \rightarrow L^{1}$ such that $P(D) \subset D$. 
The class of the functions $Q$ which satisfy (A3) is rather large. For example if $Q$ is a $C^{1}$-function and there exists an at most countable family of intervals $\left[a_{i}, b_{i}\right], i \in I$, such that

$$
[0, \infty)=\bigcup_{i \in I}\left[a_{i}, b_{i}\right], \quad\left(a_{i}, b_{i}\right) \cap\left(a_{j}, b_{j}\right)=\emptyset \quad \text { for } i \neq j
$$

and $Q^{\prime}(x) \neq 0$ for $x \in\left(a_{i}, b_{i}\right)$ and $i \in I$, then $Q$ satisfies (A3) and the operator $P_{Q}$ is given by the formula

$$
P_{Q} f(x)=\sum_{i \in I_{x}} f\left(\varphi_{i}(x)\right)\left|\varphi_{i}^{\prime}(x)\right|,
$$

where $\varphi_{i}$ is the inverse function of $\left.Q\right|_{\left(a_{i}, b_{i}\right)}$ and $I_{x}=\left\{i: \varphi_{i}(x) \in\left(a_{i}, b_{i}\right)\right\}$.

Now we add the second ingredient to the model. If $f$ is the initial density of immune status and there is no infection till the time $t$, then the density of immune status at $t$ is given by $S(t) f$, where $S(t)$ is the Frobenius-Perron operator related to the transformation $x \mapsto \pi_{t} x$. In this way we obtain a $C_{0}$-semigroup of stochastic operators $\{S(t)\}_{t \geq 0}$ given by

$$
S(t) f(x)= \begin{cases}f\left(\pi_{-t} x\right) \frac{\partial\left(\pi_{-t} x\right)}{\partial x} & \text { if } \pi_{-t} x \text { exists, } \\ 0 & \text { if } \pi_{-t} x \text { does not exist. }\end{cases}
$$

The semigroup $\{S(t)\}_{t \geq 0}$ has the infinitesimal generator $\mathcal{A}_{0} f(x)=-\frac{d}{d x}(g(x) f(x))$ with the domain $\mathfrak{D}\left(\mathcal{A}_{0}\right)=\left\{f \in L^{1}: \mathcal{A}_{0} f \in L^{1}\right\}$. Here the notation $\mathcal{A}_{0} f \in L^{1}$ means that $f$ is a locally absolutely continuous function, so $f^{\prime}$ exists a.e., and $(g f)^{\prime} \in L^{1}$. The adjoint semigroup $\left\{S^{*}(t)\right\}_{t \geq 0}$ on $L^{\infty}$ is given by the formula $S^{*}(t) f(x)=f\left(\pi_{t} x\right)$.

Finally, we combine both ingredients: waning and boosting of immunity status. Then the density $u(t)=u(t, x)$ of immune status satisfies the following evolution equation in $L^{1}$

$$
u^{\prime}(t)=\mathcal{A} u(t),
$$

where $\mathcal{A}=\mathcal{A}_{0}+\Lambda P_{Q}-\Lambda I$. The solution $u(t)$ of this equation generates a stochastic semigroup (i.e. a $C_{0}$-semigroup of stochastic operators) $\{U(t)\}_{t \geq 0}$. It means that if $f$ is the density of initial immune status then $U(t) f$ is the density of immune status at time $t$. The semigroup $\{U(t)\}_{t \geq 0}$ is given by the Dyson-Phillips expansion

$$
U(t) f=e^{-\Lambda t} \sum_{n=0}^{\infty} \Lambda^{n} S_{n}(t) f
$$


where

$$
S_{0}(t) f=S(t) f, \quad S_{n+1}(t) f=\int_{0}^{t} S_{n}(t-\tau) P_{Q} S_{0}(\tau) f d \tau, \quad n \geq 0 .
$$

Similar formulas to (5)-(6) hold for the adjoint semigroup $\left\{U^{*}(t)\right\}_{t \geq 0}$ on $L^{\infty}$. In particular if $f \geq 0$ then

$$
U^{*}(t) f \geq \Lambda e^{-\Lambda t} \int_{0}^{t} S^{*}(t-\tau) P_{Q}^{*} S^{*}(\tau) f d \tau=\Lambda e^{-\Lambda t} \int_{0}^{t} f\left(\pi_{\tau} Q\left(\pi_{t-\tau} x\right)\right) d \tau .
$$

The process $\left(\xi_{t}\right)_{t \geq 0}$ has the probability transition function $\mathcal{P}(t, x, \Gamma)$ given by

$$
\mathcal{P}(t, x, \Gamma)=U^{*}(t) \mathbf{1}_{\Gamma}(x) .
$$

Now inequality (7) allows us to estimate $\mathcal{P}(t, x, \Gamma)$ from below

$$
\mathcal{P}(t, x, \Gamma) \geq \Lambda e^{-\Lambda t} \int_{0}^{t} \mathbf{1}_{\Gamma}\left(\pi_{\tau} Q\left(\pi_{t-\tau} x\right)\right) d \tau .
$$

This inequality will play the crucial role in the proof of the existence and asymptotic stability of a stationary density.

\section{Asymptotic Stability AND SWEeping}

We start with some general definitions and results concerning asymptotic stability and sweeping of stochastic semigroups.

Let a triple $(X, \Sigma, \mu)$ be a $\sigma$-finite measure space. A stochastic semigroup $\{P(t)\}_{t \geq 0}$ on $L^{1}=L^{1}(X, \Sigma, \mu)$ is called asymptotically stable if there exists a density $f^{*}$ such that

$$
\lim _{t \rightarrow \infty}\left\|P(t) f-f^{*}\right\|=0 \quad \text { for } \quad f \in D .
$$

If the semigroup $\{P(t)\}_{t \geq 0}$ is generated by some evolution equation $u^{\prime}(t)=$ $A u(t)$ then the asymptotic stability of $\{P(t)\}_{t \geq 0}$ means that the stationary solution $u(t)=f^{*}$ is asymptotically stable in the sense of Lyapunov and this stability is global on the set $D$.

A stochastic semigroup $\{P(t)\}_{t \geq 0}$ is called partially integral if there exist $t>0$ and a measurable function $q(t, \cdot, \cdot): X \times X \rightarrow[0, \infty)$ such that

$$
P(t) f(y) \geq \int_{X} q(t, x, y) f(x) \mu(d y) \quad \text { for } f \in D,
$$

and

$$
\int_{X} \int_{X} q(t, x, y) \mu(d x) \mu(d y)>0 .
$$


If $\mathcal{P}(t, x, d y)$ is the transition probability function corresponding to the stochastic semigroup $\{P(t)\}_{t \geq 0}$ then inequality 10 can be written in an equivalent form $\mathcal{P}(t, x, d y) \geq q(t, x, y) d y$. We will use the following criterion of asymptotic stability.

Theorem 1. 10] Let $\{P(t)\}_{t \geq 0}$ be a partially integral stochastic semigroup. Assume that the semigroup $\{P(t)\}_{t \geq 0}$ has a unique invariant density $f^{*}$. If $f^{*}>0$ a.e., then the semigroup $\{P(t)\}_{t \geq 0}$ is asymptotically stable.

A stochastic semigroup $\{P(t)\}_{t \geq 0}$ is called sweeping with respect to a set $B \in \Sigma$ if for every $f \in D$

$$
\lim _{t \rightarrow \infty} \int_{B} P(t) f(x) \mu(d x)=0 .
$$

From now on we assume additionally that $(X, \rho)$ is a separable metric space and $\Sigma=\mathcal{B}(X)$ is the $\sigma$-algebra of Borel subsets of $X$. We will consider stochastic semigroups $\{P(t)\}_{t \geq 0}$ which satisfy the following condition:

(K) for every $x_{0} \in X$ there exist an $\varepsilon>0$, a $t>0$, and a measurable function $\eta \geq 0$ such that $\int \eta(x) \mu(d x)>0$ and

$$
\mathcal{P}(t, x, d y) \geq \eta(y) \mu(d y) \quad \text { for } x \in B\left(x_{0}, \varepsilon\right),
$$

where $B\left(x_{0}, \varepsilon\right)=\left\{x \in X: \rho\left(x, x_{0}\right)<\varepsilon\right\}$.

It is clear that if a stochastic semigroup satisfies condition $(K)$ then it is partially integral. We will need the following criterion of sweeping [11, Corollary 2].

Theorem 2. Assume that a stochastic semigroup $\{P(t)\}_{t \geq 0}$ satisfies condition $(\mathrm{K})$ and has no invariant densities. Then $\{P(t)\}_{t \geq 0}$ is sweeping from compact sets.

We say that a stochastic semigroup $\{P(t)\}_{t \geq 0}$ satisfies the Foguel alternative if it is asymptotically stable or sweeping from all compact sets [7. We now formulate the main result of this paper.

Theorem 3. The semigroup $\{U(t)\}_{t \geq 0}$ satisfies the Foguel alternative, i.e. it is asymptotically stable or for every $f \in L^{1}[0, \infty)$ and $M>0$

$$
\lim _{t \rightarrow \infty} \int_{0}^{M} U(t) f(x) d x=0 .
$$


In order to prove Theorem 2 it is enough to check that the semigroup $\{U(t)\}_{t \geq 0}$ satisfies condition $(\mathrm{K})$ and that if $f^{*}$ is an invariant density for $\{U(t)\}_{t \geq 0}$ then $f^{*}(x)>0$ a.e. Indeed, if $\{U(t)\}_{t \geq 0}$ has no invariant densities, then according to Theorem 2 this semigroup is sweeping from compact sets. In the case when $\{U(t)\}_{t \geq 0}$ has more then one invariant density then it is easy to construct two invariant densities $f_{1}^{*}$ and $f_{2}^{*}$ with disjoint supports, i.e. such that $f_{1}^{*} f_{2}^{*}=0$ a.e. Thus, the uniqueness of an invariant density will be a simple consequence of its strict positivity. It means that if an invariant density exists and we know that this density has to be positive then according to Theorem 1 the semigroup $\{U(t)\}_{t \geq 0}$ is asymptotically stable.

Lemma 1. The semigroup $\{U(t)\}_{t \geq 0}$ fulfills condition $(\mathrm{K})$.

Proof. From (8) it follows that

$$
\mathcal{P}(t, x, \Gamma) \geq \Lambda e^{-\Lambda t} \int_{0}^{t} \mathbf{1}_{\Gamma}(r(\tau, t, x)) d \tau
$$

where $r(\tau, t, x)=\pi_{\tau} Q\left(\pi_{t-\tau} x\right)$. First we want to find the derivative $\frac{\partial r}{\partial \tau}$. We use the following formulas:

$$
\frac{\partial}{\partial t}\left(\pi_{t} x\right)=g\left(\pi_{t} x\right), \quad \frac{\partial}{\partial x}\left(\pi_{t} x\right)=\frac{g\left(\pi_{t} x\right)}{g(x)} .
$$

The first formula follows directly from the definition of $\pi_{t} x$. Now we derive the second one. Let $\varphi(t, x)=\frac{\partial}{\partial x}\left(\pi_{t} x\right)$. Then

$$
\frac{\partial \varphi}{\partial t}(t, x)=\frac{\partial}{\partial x} \frac{\partial}{\partial t}\left(\pi_{t} x\right)=\frac{\partial}{\partial x} g\left(\pi_{t} x\right)=g^{\prime}\left(\pi_{t} x\right) \frac{\partial}{\partial x}\left(\pi_{t} x\right)=g^{\prime}\left(\pi_{t} x\right) \varphi(t, x) .
$$

Hence

but since $\varphi(0, x)=1$ we have

$$
\frac{\partial}{\partial t}(\ln \varphi(t, x))=g^{\prime}\left(\pi_{t} x\right)
$$

$$
\ln \varphi(t, x)=\int_{0}^{t} g^{\prime}\left(\pi_{s} x\right) d s=\int_{x}^{\pi_{t} x} \frac{g^{\prime}(y)}{g(y)} d y=\left.\ln g(y)\right|_{y=x} ^{y=\pi_{t} x}=\ln \left(\frac{g\left(\pi_{t} x\right)}{g(x)}\right),
$$

which proves the second formula of $(13)$. From the chain role we obtain

$$
\frac{\partial r}{\partial \tau}(\tau, t, x)=g\left(\pi_{\tau} Q\left(\pi_{t-\tau} x\right)\right)-\frac{g\left(\pi_{\tau} Q\left(\pi_{t-\tau} x\right)\right)}{g\left(Q\left(\pi_{t-\tau} x\right)\right)} Q^{\prime}\left(\pi_{t-\tau} x\right) g\left(\pi_{t-\tau} x\right) .
$$

If $\tau=0$ and $x=x_{0}$, then

$$
\lim _{t \rightarrow \infty} \frac{\partial r}{\partial \tau}\left(0, t, x_{0}\right)=\lim _{t \rightarrow \infty}\left[g\left(Q\left(\pi_{t} x_{0}\right)\right)-Q^{\prime}\left(\pi_{t} x_{0}\right) g\left(\pi_{t} x_{0}\right)\right]=g(Q(0)) .
$$


Since $g(Q(0))<0$ and $r$ is a $C^{1}$ function we can find a sufficiently large $t$ and positive constants $\varepsilon^{\prime}, \delta, M$, and $\tau_{0} \leq t$ such that

$$
-M \leq \frac{\partial r}{\partial \tau}(\tau, t, x) \leq-\delta \text { for } \tau \in\left[0, \tau_{0}\right] \text { and } x \in B\left(x_{0}, \varepsilon^{\prime}\right) .
$$

From $(12)$ it follows that

$$
\mathcal{P}(t, x, \Gamma) \geq \Lambda e^{-\Lambda t} \int_{0}^{\tau_{0}} \mathbf{1}_{\Gamma}(r(\tau, t, x)) d \tau \geq \frac{\Lambda e^{-\Lambda t}}{M} \int_{\Delta_{x}} \mathbf{1}_{\Gamma}(y) d y
$$

for $x \in B\left(x_{0}, \varepsilon^{\prime}\right)$, where $\Delta_{x}=\left[r\left(\tau_{0}, t, x\right), r(0, t, x)\right]$. The interval $\Delta_{x}$ has the length at least $\delta \tau_{0}$. Let $\varepsilon \in\left(0, \varepsilon^{\prime}\right)$ be such that

$$
\left|r(0, t, x)-r\left(0, t, x_{0}\right)\right|<\delta \tau_{0} / 3 \quad \text { for } x \in B\left(x_{0}, \varepsilon\right) .
$$

Then we find an interval $\Delta$ with a length of at least $\delta \tau_{0} / 3$ such that $\Delta \subset \Delta_{x}$ for $x \in B\left(x_{0}, \varepsilon\right)$. Let $\eta(y)=\Lambda e^{-\Lambda t} M^{-1} \mathbf{1}_{\Delta}(y)$. Then $\mathcal{P}(t, x, d y) \geq \eta(y) d y$ for $x \in B\left(x_{0}, \varepsilon\right)$.

Lemma 2. If $f^{*}$ is an invariant density with respect to $\{U(t)\}_{t \geq 0}$, then $f^{*}>0$ a.e.

Proof. Let $A=\left\{x: f^{*}(x)>0\right\}$. The set $A$ is defined up to a set of measure zero. Since

$$
f^{*}(x)=U(t) f^{*}(x) \geq e^{-\Lambda t} S(t) f^{*}(x)=e^{-\Lambda t} f^{*}\left(\pi_{-t} x\right) \frac{\partial \pi_{-t} x}{\partial x}>0
$$

for $x \in \pi_{t}(A)$ and $t \geq 0$, we have $\pi_{t}(A) \subseteq A$ for arbitrary $t>0$, and consequently $A=(0, a)$ or $A=(0, \infty)$. We check that $A=(0, \infty)$. Assume on the contrary that $A=(0, a)$. Then $S(\tau) f^{*}(x)>0$ for $x \in(0, b), b=$ $\pi_{\tau}(a)$. Let $m=\min \{Q(x): x \geq 0\}$ and assume that $Q(a) \neq m$. Observe that if $f(x)>0$ for $x \in(0, b)$, then $P_{Q} f(x)>0$ for all $x \in(m, Q(b))$. It means that

$$
S(t-\tau) P_{Q} S(\tau) f^{*}(x)>0 \quad \text { for } x \in\left(\pi_{t-\tau} m, \pi_{t-\tau} Q\left(\pi_{\tau}(a)\right)\right) .
$$

Since

$$
f^{*}(x) \geq \Lambda e^{-\Lambda t} S_{1}(t) f^{*}(x)=\Lambda e^{-\Lambda t} \int_{0}^{t} S(t-\tau) P_{Q} S(\tau) f^{*}(x) d \tau,
$$

we have $f^{*}(x)>0$ for $x \in\left(\pi_{t} m, \pi_{t} Q(a)\right)$. As $m<Q(a)$, the interval $I_{t}=\left(\pi_{t} m, \pi_{t} Q(a)\right)$ is nontrivial. Moreover, $\pi_{t} Q(a)>a$ for sufficiently small $t>0$, which contradicts the definition of $A$. In the case $Q(a)=m$ we need an extra argument. From assumption (A3) it follows that the transformation $Q$ 
cannot be constant on any nontrivial interval. Let $\bar{m}=\max \{Q(x): x \leq a\}$. If $f(x)=P_{Q} S(\tau) f^{*}(x)$, then $f(x)>0$ for $x \in Q\left(\left(0, \pi_{\tau} a\right)\right)$. We can find an $\varepsilon>0$ such that $[\bar{m}-\varepsilon, \bar{m}] \subset Q\left(\left(0, \pi_{\tau} a\right)\right)$ for sufficiently small $\tau>0$. Hence $S(t-\tau) P_{Q} S(\tau) f^{*}(x)>0$ for $x \in J_{t}$ where $J_{t}=\left(\pi_{t-\tau}(\bar{m}-\varepsilon), \pi_{t-\tau} \bar{m}\right)$. Using the same argument as in the previous case we check that $f^{*}(x)>0$ for $x \in J_{t}$. Finally, the inequality $\pi_{t-\tau} \bar{m}>m$ for sufficiently small $t$ implies that $J_{t} \not \subset A$, which contradicts the definition of $A$.

Proof of Theorem 3. Theorem 3 is a simple consequence of Theorems 1, 2 and Lemmas 1, 2,

Assumptions (A1)-(A3) are not sufficient to prove asymptotic stability of the semigroup $\{U(t)\}_{t \geq 0}$, but according to Theorem 3 we only need to check when the semigroup $\{U(t)\}_{t \geq 0}$ is weakly tight, i.e. there exists $\kappa>0$ such that

$$
\sup _{F \in \mathcal{F}} \limsup _{t \rightarrow \infty} \int_{F} U(t) f(x) d x \geq \kappa
$$

for $f \in D_{0}$, where $D_{0}$ is a dense subset of $D$ and $\mathcal{F}$ is the family of all compact subsets of $X$. It is clear that weak tightness excludes the case when the semigroup is sweeping from compact sets. The process $\left(\xi_{t}\right)_{t \geq 0}$ has the infinitesimal generator

$$
\mathcal{L} V(x)=g(x) V^{\prime}(x)+\Lambda V(Q(x))-\Lambda V(x) .
$$

The operators $\mathcal{A}$ and $\mathcal{L}$ are formally conjugated, i.e.

$$
\int_{0}^{\infty} \mathcal{A} f(x) h(x) d x=\int_{0}^{\infty} f(x) \mathcal{L} h(x) d x \quad \text { for } f \in \mathfrak{D}(\mathcal{A}) \text { and } h \in \mathfrak{D}(\mathcal{L}) .
$$

Assume that there exist a $C^{1}$-function $V:[0, \infty) \rightarrow[0, \infty)$ and constants $\varepsilon, r, \bar{M}>0$ such that

$$
\mathcal{L} V(x) \leq \bar{M} \text { for } x<r \quad \text { and } \quad \mathcal{L} V(x) \leq-\varepsilon \quad \text { for } x \geq r .
$$

Then the semigroup $\{U(t)\}_{t \geq 0}$ is weakly tight (see e.g. [15, page 128] for a general result).

Since $V, Q, g$ are $C^{1}$-functions, the inequality $\mathcal{L} V(x) \leq \bar{M}$ for $x<r$ is obviously fulfilled. Therefore it remains to check when there exists a $C^{1}$ function $V:[0, \infty) \rightarrow[0, \infty)$ such that

$$
\limsup _{x \rightarrow \infty}\left[g(x) V^{\prime}(x)+\Lambda V(Q(x))-\Lambda V(x)\right]<0 .
$$


For example, assume that the immune status is roughly proportional to the concentration of antibodies and their degradation rate is almost constant, then $\lim _{x \rightarrow \infty} g(x)=-\infty$. Also assume that the increase of the concentration of antibodies after the infection is bounded, i.e. $Q(x) \leq x+L$, then condition (18) is fulfilled with the function $V(x)=x$. It means that the semigroup $\{U(t)\}_{t \geq 0}$ is asymptotically stable.

Condition (18) also holds under much less restrictive assumptions. For example if $g(x) \leq-a x$ and $Q(x) \leq b x$ for a sufficiently large $x$, we can take $V(x)=x^{\gamma}, \gamma>0$, and check when

$$
-a \gamma+\Lambda b^{\gamma}-\Lambda<0
$$

If $a>\Lambda \log b$, then taking a sufficiently small $\gamma$ we obtain (18).

If $a<\Lambda \log b, g(x) \leq-a x$ and $Q(x) \geq b x$ then the semigroup is sweeping from compact sets. Indeed, consider a negative moment of the process $\left(\xi_{t}\right)_{t \geq 0}$

$$
m_{\gamma}(t)=\mathrm{E} \xi_{t}^{-\gamma}=\int_{0}^{\infty} x^{-\gamma} u(t, x) d x
$$

One can easy check that

$$
\frac{d}{d t} m_{\gamma}(t) \leq c_{\Lambda} m_{\gamma}(t)
$$

where $c_{\gamma}=\gamma a+\Lambda b^{-\gamma}-\Lambda$. Assume that $\mathrm{E} \xi_{0}^{-\gamma}<\infty$ (this inequality is fulfilled for example if $\xi_{0}$ takes values from some interval $\left.[\alpha, \beta], 0<\alpha<\beta<\infty\right)$. We have $c_{\gamma}<0$ for a sufficiently small $\gamma$, and consequently $\lim _{t \rightarrow \infty} m_{\gamma}(t)=0$. But in this case the semigroup $\{U(t)\}_{t \geq 0}$ is not asymptotically stable and, in consequence, $\{U(t)\}_{t \geq 0}$ is sweeping from compact sets.

Remark 1. Theorem 3 can be formulated in a slightly stronger form. Denote by $\nu_{t}$ the distribution of the process $\left(\xi_{t}\right)_{t \geq 0}$ at time $t$. We do not assume now that the measure $\nu_{0}$ has a density. Consider the case when there is an invariant density $f^{*}$. Let $\nu^{*}$ be the measure with density $f^{*}$. Then the measures $\nu_{t}$ converge to the measure $\nu^{*}$ in the total variation norm. This result follows from the fact that if $\nu_{t}^{s}$ is the singular part of the measure $\nu_{t}$, then $\lim _{t \rightarrow \infty} \nu_{t}^{s}([0, \infty))=0$. 


\section{Models With Bounded phase SPaCeS}

Now we consider the case when the immune status is a number from the interval $X=[0, M]$. We start with a version of the model introduced in Section 2, We assume that

(B1) $g:[0, M] \rightarrow \mathbb{R}$ is a $C^{1}$-function such that $g(x)<0$ for $x>0$ and $g(0)=0$,

(B2) $Q:[0, M] \rightarrow(0, M]$ is a $C^{1}$-function such that $Q(x)>x$ for $x \in$ $[0, M)$ and $Q(M)=M$,

(B3) $|A|=0 \Longrightarrow\left|Q^{-1}(A)\right|=0$, where $A$ is a Borel subset of $[0, M]$.

Then in the same way as in the previous sections we introduce a stochastic semigroup $\{U(t)\}_{t \geq 0}$ on the space $L^{1}(X, \mathcal{B}(X),|\cdot|)$ and prove an appropriate version of Theorem 3. But now $X$ is a compact space and, in consequence, the semigroup $\{U(t)\}_{t \geq 0}$ is not sweeping from compact sets. Therefore we can formulate the following

Theorem 4. The semigroup $\{U(t)\}_{t \geq 0}$ is asymptotically stable.

Now we consider a model with an alternative version of the function $Q$ proposed in [4]. We replace assumptions (B2) and (B3) by the following

$\left(\mathrm{B} 2^{\prime}\right)$ there exists $K \in(0, M)$ such that $Q:[0, K) \rightarrow(0, M]$ is a $C^{1}$ function such that $x<Q(x)<M$ for $x \in[0, K)$ and $Q(x)=M$ for $x \in[K, M]$,

$\left(\mathrm{B}^{\prime}\right)|A|=0 \Longrightarrow\left|Q^{-1}(A)\right|=0$, where $A$ is a Borel subset of $[0, M)$.

Illustrative examples of graphs of the transformation $Q$ for both considered cases are given in Fig. 2 ,

Observe that in this case the transformation $Q$ does not satisfy condition (B3). Indeed, if $A=\{M\}$ then $|A|=0$ but the set $Q^{-1}(A)=[K, M]$ has a positive Lebesgue measure. It means that we cannot define the FrobeniusPerron operator $P_{Q}$ on the space $L^{1}(X, \mathcal{B}(X),|\cdot|)$. In order to use introduced earlier apparatus of stochastic semigroups we need to modify the definition of the infinitesimal generator $\mathcal{A}$ of the semigroup $\{U(t)\}_{t \geq 0}$. The starting point can be the infinitesimal generator $\mathcal{L}$ of the process $\left(\xi_{t}\right)_{t \geq 0}$ given by $(16)$ and we want to find the operator $\mathcal{A}$ as a formally adjoint operator of $\mathcal{L}$. First, we define some modification of the Frobenius-Perron operator. If $\widetilde{Q}=\left.Q\right|_{[0, K)}$, 

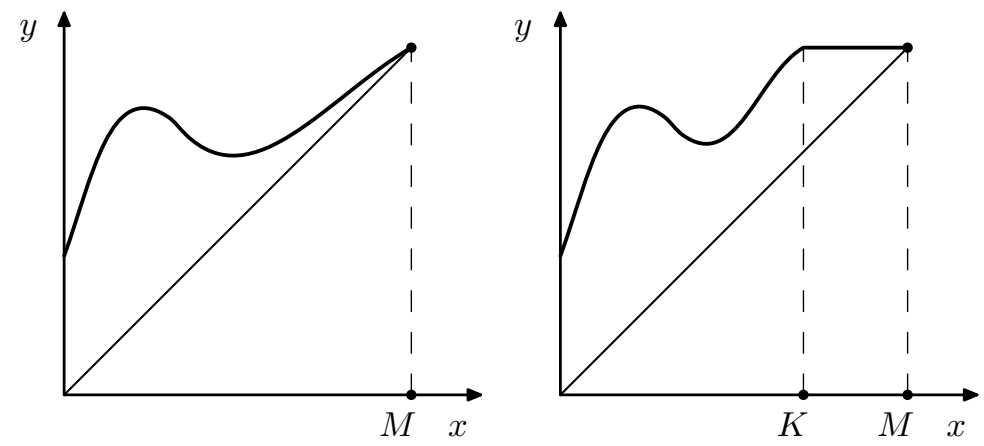

Figure 2. Examples of graphs of $y=Q(x)$. Left: condition (B2); right: condition $\left(\mathrm{B}^{\prime}\right)$.

then according to $\left(\mathrm{B}^{\prime}\right)$ we can define an operator $P_{\widetilde{Q}}: L^{1}[0, K] \rightarrow L^{1}[0, M]$ by formula (2). Then $P_{\widetilde{Q}}$ is a stochastic operator in the sense that it is a linear transformation from $L^{1}[0, K]$ to $L^{1}[0, M]$ and $P_{\widetilde{Q}}$ maps densities to densities. Next we define the operator $\bar{P}_{\widetilde{Q}}: L^{1}[0, M] \rightarrow L^{1}[0, M]$ by $\bar{P}_{\widetilde{Q}} f=$ $P_{\widetilde{Q}}\left(\left.f\right|_{[0, K)}\right)$. Then $\bar{P}_{\widetilde{Q}}$ is a substochastic operator, i.e. $\bar{P}_{\widetilde{Q}}$ is a positive contraction of $L^{1}$. The operator $\mathcal{A}$ is defined on the set

$\mathfrak{D}(\mathcal{A})=\left\{f \in L^{1}[0, M]: f^{\prime} \in L^{1}[0, M]\right.$ and $\left.g(M) f(M)=-\Lambda \int_{K}^{M} f(x) d x\right\}$ and $\mathcal{A}$ is given by

$$
\mathcal{A} f=-(g f)^{\prime}+\Lambda \bar{P}_{\widetilde{Q}} f-\Lambda f .
$$

It is not difficult to check that the operators $\mathcal{A}$ and $\mathcal{L}$ are formally conjugated.

Now we can write the evolution of densities of the process $\left(\xi_{t}\right)_{t \geq 0}$ in the form of the abstract Cauchy problem (4). We can treat Eq. (4) as abstract notation of a first order partial differential equation with some linear perturbation and some boundary condition. Such equations appear in many biological and physical applications, e.g. in structured population models [2, 8, 9, 13].

One can check that the operator $\mathcal{A}$ generates a stochastic semigroup on the space $L^{1}(X, \mathcal{B}(X),|\cdot|)$. The proof of this result is rather standard so we only sketch it omitting the computational part. Some new and general results concerning piecewise deterministic Markov processes with boundary can be found in [6]. 
We start with some definitions and two general results concerning generators of substochastic and stochastic semigroups. Let $A$ be a linear operator defined on a linear subspace $\mathfrak{D}(A)$ of a Banach space $E$. We say that $\lambda \in \mathbb{R}$ belongs to the resolvent set $\rho(A)$ of $A$, if the operator $\lambda I-A: \mathfrak{D}(A) \rightarrow E$ is invertible. The operator $\mathcal{R}(\lambda, A):=(\lambda I-A)^{-1}$ for $\lambda \in \rho(A)$ is called the resolvent operator of $A$ at $\lambda$. Now let $E=L^{1}(X, \Sigma, \mu)$. We call a linear operator $A$ resolvent positive if there exists $\omega \in \mathbb{R}$ such that $(\omega, \infty) \subseteq \rho(A)$ and $\mathcal{R}(\lambda, A) \geq 0$ for all $\lambda>\omega$. Let $L_{+}^{1}=\left\{f \in L^{1}: f \geq 0\right\}$ and $\mathfrak{D}(A)_{+}=$ $\mathfrak{D}(A) \cap L_{+}^{1}$. A $C_{0}$-semigroup of substochastic operators on the space $L^{1}$ is called shortly a substochastic semigroup.

Theorem 5. A linear operator $A$ with the domain $\mathfrak{D}(A) \subset L^{1}$ is the generator of a substochastic semigroup on $L^{1}$ if and only if $\mathfrak{D}(A)$ is dense in $L^{1}$, the operator $A$ is resolvent positive, and

$$
\int_{X} A f(x) \mu(d x) \leq 0 \quad \text { for all } f \in \mathfrak{D}(A)_{+} .
$$

The proof of this result is given e.g. in [15, Theorem 4.4]. The second result concerns positive perturbations of substochastic semigroups [1, Section $6.2]$.

Theorem 6. Assume that the operator $A_{0}$ is the generator of a substochastic semigroup $\{S(t)\}_{t \geq 0}$ on $L^{1}$ and $B$ is a positive and bounded operator on $L^{1}$ such that

$$
\int_{X}\left(A_{0} f(x)+B f(x)\right) \mu(d x)=0 \quad \text { for } \quad f \in \mathfrak{D}\left(A_{0}\right)_{+} .
$$

Then the operator $A=A_{0}+B$ is the generator of a stochastic semigroup $\{U(t)\}_{t \geq 0}$ on $L^{1}$.

Now we apply Theorems 5 and 6 to the operator $\mathcal{A}$. Consider the operator $\mathcal{A}_{0} f=-(g f)^{\prime}-\Lambda f$ with the domain $\mathfrak{D}\left(\mathcal{A}_{0}\right)=\mathfrak{D}(\mathcal{A})$ and the operator $\mathcal{B} f=$ $\Lambda \bar{P}_{\widetilde{Q}} f$. Then $\mathcal{B}$ is a positive and bounded operator on the space $L^{1}$. The operator $\mathcal{A}_{0}$ generates a substochastic semigroup $\{S(t)\}_{t \geq 0}$ on the space $L^{1}$. This statement is intuitively obvious because the equation $u^{\prime}(t)=\mathcal{A}_{0} u(t)$ describes the movement of particles to the left on the interval $[0, M]$ with the influx of new particles through the right end $M$ with velocity $\int_{K}^{M} u(t, x) d x$ and the efflux from the interval $[0, M]$ with velocity $\int_{0}^{M} u(t, x) d x$. The proof that the operator $\mathcal{A}_{0}$ generates a substochastic semigroup follows from 
Theorem 5. It is easy to check that $\mathfrak{D}\left(\mathcal{A}_{0}\right)$ is a dense subset of $L^{1}$ and that condition 190 holds. Then we find that

$$
\mathcal{R}\left(\lambda, \mathcal{A}_{0}\right) f(x)=-\frac{e^{(\lambda+\Lambda) \varphi(x)}}{g(x)}\left(\Lambda I(\lambda, f)+\int_{x}^{M} f(r) e^{-(\lambda+\Lambda) \varphi(r)} d r\right),
$$

where $\varphi(x)=\int_{x}^{M} \frac{d r}{g(r)}$ and $I(\lambda, f)$ is a constant such that

$$
I(\lambda, f)=\int_{K}^{M} \mathcal{R}\left(\lambda, \mathcal{A}_{0}\right) f(x) d x .
$$

It is also easy to observe that $\mathcal{R}\left(\lambda, \mathcal{A}_{0}\right) \geq 0$ for all $\lambda>0$. Since

$$
\begin{aligned}
\int_{0}^{M} & \left(\mathcal{A}_{0} f(x)+\mathcal{B} f(x)\right) d x=\int_{0}^{M}\left(-(g f)^{\prime}(x)-\Lambda f(x)+\Lambda \bar{P}_{\widetilde{Q}} f(x)\right) d x \\
= & -g(M) f(M)-\Lambda \int_{0}^{M} f(x) d x+\Lambda \int_{0}^{M} P_{\widetilde{Q}}\left(\left.f\right|_{[0, K)}\right)(x) d x \\
= & \Lambda \int_{K}^{M} f(x) d x-\Lambda \int_{0}^{M} f(x) d x+\Lambda \int_{0}^{K} f(x) d x=0,
\end{aligned}
$$

according to Theorem 6 the semigroup $\{U(t)\}_{t \geq 0}$ generated by the operator $\mathcal{A}$ is a stochastic semigroup.

Theorem 4 remains true in this case. The only difference in the proof is that instead of formulas (5)-(6) we need to apply the two following ones

$$
U(t) f=\sum_{n=0}^{\infty} S_{n}(t) f,
$$

where

$$
S_{0}(t) f=S(t) f, \quad S_{n+1}(t) f=\int_{0}^{t} S_{n}(t-\tau) \mathcal{B} S_{0}(\tau) f d \tau, \quad n \geq 0 .
$$

\section{REFERENCES}

[1] Banasiak J, Arlotti L. Perturbations of Positive Semigroups with Applications. London: Springer Monographs in Mathematics. Springer-Verlag; 2006.

[2] Banasiak J, Pichór K, Rudnicki R. Asynchronous exponential growth of a general structured population model. Acta Appl. Math. 2012;119:149-166.

[3] Davis MHA. Piecewise-deterministic Markov processes: A general class of nondiffusion stochastic models. J. Roy. Statist. Soc. Ser. B 1984;46:353-388.

[4] Diekmann O, de Graaf WF, Kretzschmar MEE, Teunis PFM. Waning and boosting: on the dynamics of immune status. J. Math. Biol. 2018;77:2023-2048.

[5] de Graaf WF, Kretzschmar MEE, Teunis PFM, Diekmann O. A two-phase withinhost model for immune response and its application to serological profiles of pertussis. Epidemics 2014;9:1-7.

[6] Gwiżdż P, Tyran-Kamińska M. Densities for piecewise deterministic Markov processes with boundary. J. Math. Anal. Appl. 2019;479:384-425.

[7] Lasota A, Mackey MC. Chaos, Fractals and Noise. Stochastic Aspects of Dynamics. New York: Springer Applied Mathematical Sciences vol. 97 Springer; 1994. 
[8] Mackey MC, Rudnicki R. Global stability in a delayed partial differential equation describing cellular replication. J. Math. Biol. 1994;33:89-109.

[9] Mackey MC, Tyran-Kamińska M. Dynamics and density evolution in piecewise deterministic growth processes. Ann. Polon. Math. 2008;94:111-129.

[10] Pichór K, Rudnicki R. Continuous Markov semigroups and stability of transport equations. J. Math. Anal. Appl. 2000;249:668-685.

[11] Pichór K, Rudnicki R. Asymptotic decomposition of substochastic operators and semigroups. J. Math. Anal. Appl. 2016;436:305-321.

[12] Pichór K, Rudnicki R. Asymptotic decomposition of substochastic semigroups and applications. Stoch. Dynam. 2018;18:1850001, 18 pp.

[13] Pichór K, Rudnicki R. Applications of stochastic semigroups to cell cycle models. Discrete Contin. Dyn. Syst. Ser. B 2019;24:2365-2381.

[14] Rudnicki R. Stochastic operators and semigroups and their applications in physics and biology. In: Banasiak J, Mokhtar-Kharroubi M, eds. Evolutionary Equations with Applications in Natural Sciences, Lecture Notes in Mathematics, vol. 2126: Heidelberg: Springer 2015 (pp. 255-318).

[15] Rudnicki R, Tyran-Kamińska M. Piecewise Deterministic Processes in Biological Models. Cham, Switzerland: SpringerBriefs in Applied Sciences and Technology, Mathematical Methods, Springer; 2017.

[16] Teunis PFM, van Eijkeren JCH, de Graaf WF, Bonačić Marinović A, Kretzschmar MEE. Linking the seroresponse to infection to within-host heterogeneity in antibody production. Epidemics 2016;16:33-39.

K. Pichór, Institute of Mathematics, University of Silesia, Bankowa 14, 40-007 Katowice, Poland.

E-mail address: katarzyna.pichor@us.edu.pl

R. Rudnicki, Institute of Mathematics, Polish Academy of Sciences, Bankowa 14, 40-007 Katowice, Poland.

E-mail address: rudnicki@us.edu.pl 